syndrome. A study comparing cognitive profiles of children aged 7-16 years with MS $(n=9)$ to those with ADEM $(n=9)$ found impairments across all cognitive domains with differences in severity and spread. The transient white matter disruption in ADEM results in subtle cognitive impairments, while the multiple white matter insults in childhood MS are associated with more severe cognitive sequelae (Deery B et al. Dev Neuropsychol 2010;35(5):506-521).

\title{
CONGENITAL MALFORMATIONS
}

\section{MAJOR BIRTH DEFECTS AND NEWER ANTIEPILEPTIC DRUGS}

The association between fetal exposure to newer-generation antiepileptic drugs during the first trimester of pregnancy and the risk of major birth defects was studied by researchers at the Statens Serum Institut, Copenhagen, Denmark. Cases of birth defects diagnosed within the first year of life following fetal exposure to AEDs were identified through the National Patient Registry, Jan 1996 through March 2009. Dispensed AEDs to mothers were ascertained from nationwide registries. Of 1532 infants exposed to lamotrigine, oxcarbazepine, topiramate, gabapentin, or levetiracetam during the first trimester, $49(3.2 \%)$ were diagnosed with a major birth defect compared to $2.4 \%$ of unexposed infants. The prevalence of major birth defects following exposure to individual AEDs was $3.7 \%$ following lamotrigine, $2.8 \%$ with oxcarbazepine, $4.6 \%$ with topiramate, $1.7 \%$ with gabapentin, and 0 following levetiracetam. First-trimester exposure to newer-generation AEDs compared to no exposure was not associated with an increased risk of major birth defects diagnosed in the first year of life. (Molgaard-Nielsen D, Hviid A. Newer-generation antiepileptic drugs and the risk of major birth defects. JAMA May 18, 2011;305(19):1996-2002). (Response: Ditte Molgaard-Nielsen, MSc, Artillerivej 5, 2300 Copernhagen 5, Denmark.. E-mail: dnl@ssi.dk).

COMMENT. The authors comment that unadjusted estimates did show a significant association between exposure to any newer AED or lamotrigine alone during the first trimester and risk of major birth defects. A significantly increased risk of eye defects was observed for lamotrigine, but only 4 infants were exposed and affected. After adjustment for older-generation AED use and epilepsy, no associations remained. Oldergeneration AEDs are associated with a 2- to 3-fold increased risk of major birth defects, and some of the mothers were treated with both older and newer generation AEDs. In this study, maternal epilepsy was associated with a moderately increased risk of birth defects and was regarded as a confounder. A meta-analysis shows that untreated women with epilepsy are not at increased risk of having infants with birth defects compared with healthy women (Fried S et al, 2004).

\section{MALFORMATIONS AFTER FETAL EXPOSURE TO AED IN UTERO ASSESSED AT BIRTH AND 12 MONTHS LATER}

Researchers at Universities of Melbourne and Queensland, Australia, compared the incidence and natures of fetal malformations (FMs) recognized at birth with those 
recognized in the first post-natal year. Up to $25 \%$ of malformations recognized by the end of the first year had not been detected by, or soon after, birth. Within a month of delivery, 64 of the 1245 pregnancies $(5.1 \%)$ and 69 of the 1268 fetuses $(5.4 \%)$ had involved FMs. By the end of the post-natal year, 85 of the pregnancies $(6.8 \%)$ and 90 of the fetuses $(7.1 \%)$ involved FMs. Excluding cases born $<1$ year before time of data analysis, the rates of occurrence of FMs were $7.4 \%$ and $7.7 \%$, respectively. The additional pregnancies involving fetal malformations recognized by 1 -year post-partum represented 21 of all the 85 pregnancies with malformations $(24.7 \%)$ and 21 of the 90 fetuses with malformations $(23.3 \%)$. Of 13 pregnancies aborted because of malformations, 8 were exposed to AED monotherapy, as valproate in 5 , lamotrigine in 2 , carbamazepine in 1 ; in 5 exposure occurred as AED polytherapy, including VPA in 5, LTG in 2, topiramate in 1, and acetazolamide in 1. Early and late assessments of FM are complementary, but omission of an early assessment may result in biases and loss of subjects to follow-up. (Vajda FJE, Graham J, Hitchcock AA, O'Brien TJ, Lander CM, Eadie MJ. Foetal malformations after exposure to antiepileptic drugs in utero assessed at birth and 12 months later: observations from the Australian pregnancy register. Acta Neurol Scand July 2011;124:9-12). (Response: FJE Vajda, Department of Medicine and Neurosciences, Royal Melbourne Hospital and University of Melbourne, Parkville 3050, Australia. Email: vajda(anetspace.net.au).

COMMENT. Previous studies of late recognition of fetal malformations (FM) have compared frequencies at birth and several years later, with similar findings (Annegers et al 1978, cited by authors). Drugs associated with FM differed between the early abnormalities and the late detected. VPA-associated malformations were detected at birth, while carbamazepine and lamotrigine were involved in those detected late. Spina bifida with hydrocephalus and cleft lip and palate were recognized at birth, while skull abnormalities were detected late. For an accurate assessment, FM rates with AED therapy during pregnancy should be checked at or soon after birth and again at 6-12 months later.

\section{HEADACHE DISORDERS}

\section{CLINICAL/MRI CHARACTERISTICS OF ACUTE MIGRAINOUS INFARCTION}

Clinical and MRI characteristics in 17 patients with acute migrainous infarction were assessed by researchers at the University of Heidelberg, Mannheim, Germany. Mean age was $44.6+/-15.9$ years, range $20-71$ years, 4 male and 13 female. All had undergone a stroke workup including diffusion-weighted imaging (DWI) between 2 hours and 7 days after onset. DWI lesions affected the posterior circulation in the majority $(70.6 \%)$, and $29.4 \%$ had middle cerebral artery infarction. Multiple lesions were found in $41.2 \%$. MRA was normal in 5 patients; in 4 the artery involving the ischemic territory showed reduced flow. Chronic white matter lesions were demonstrated in 3 cases. Patent foramen ovale was detected in $64.7 \%$. One other risk factor for ischemic stroke was present in $94.1 \%$ patients: arterial hypertension in $47.1 \%$, contraceptives $41.2 \%$, nicotine abuse $(35.2 \%$ ), and hyperlipidemia in $35.2 \%$. Coagulation abnormalities occurred in 2 patients. Presentation at the ED varied between $30 \mathrm{~min}$ and 5 days after 